\title{
From mind to molecule
}

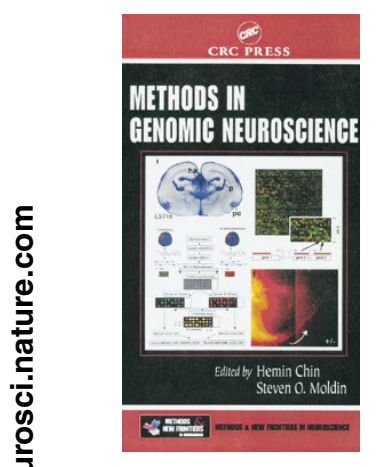

\section{Methods in Genomic Neuroscience}

edited by Hemin Chin and Steven O. Moldin

CRC Press, Boca Raton, Florida, 2002 \$119.95

ISBN: 0-84-932397-5

Reviewed by Peter Mombaerts

The nervous system differs in two fundamental ways from other organ systems. First, it is extraordinarily heterogeneous in cellular composition. Grinding up whole brain tissue to explore its inner workings is like smashing a fine Swiss watch with a hammer to understand the art of watch making. Second, its output-behavior-is also complex, and both indirect and general effects of experimental manipulations must be considered. For instance, analyzing mice with a knockout mutation in a widely expressed gene by testing how fast they swim to a hidden platform in a water maze is no longer regarded as a meaningful contribution to our knowledge of brain function. For these and other reasons, molecular and genetic studies of the nervous system require special and refined approaches.

Moreover, now that the genomic sequences of nematode, fruit fly, human and mouse are essentially complete, researchers are finally freed from the onus of studying one gene at a time in these complex organisms. Quite the opposite, actually, will soon happen; traditional gene-by-gene approaches are rapidly falling out of grace. This is particularly relevant for research into the molecular mechanisms of brain function, as the relationship between a single gene and behavior is never linear and simple. Although often the case only a few years ago, the 'one gene-one graduate student' model, and even worse, the 'one gene-one lab' model, are rapidly fading. Instead, new strategies with bewildering names such as 'massively parallel genomics' are providing a paradigm shift in molecular biology and genetics. As the nervous system has

Peter Mombaerts is at The Rockefeller University, 1230 York Avenue, New York, New York 10021, USA.

e-mail:peter@rockefeller.edu the greatest diversity of gene expression and a significant fraction of the genome is expressed exclusively in neurons, such comprehensive approaches are particularly welcome in neurobiology.

In Methods in Genomic Neuroscience, Hemin Chin and Steven Moldin, two research administrators at the National Institute of Mental Health, have invited mostly US-based neuroscientists to contribute 13 chapters that cover a wide range of molecular, genetic and genomic approaches to neurobiology. In contrast to what the title may suggest, this is not a compendium of ready-to-use laboratory protocols. Most contributions are conceptual and synthetic in nature, written in the form of a narrative. This is a wise editorial strategy, as a multi-author practical handbook is guaranteed to be seriously outdated by the time it is published. Periodicals and the internet deal much better with the fluidity and transient value of how-to information.

The volume is divided into five, untitled sections. The first three chapters deal with gene-environment interactions, naturally occurring neurological mouse mutants, and conditional and inducible targeting of genes expressed in the nervous system. The next chapters cover functional gene identification, gene trapping and chemical mutagenesis. DNA microarrays are discussed in two chapters, followed by full-length cDNA libraries, gene delivery to neural tissue and neural stem cells. The last section deals with statistical methods to analyze neurological and psychiatric disorders and traits. Although nematodes and fruit flies are anything but 'genomically challenged' organisms, little or no specific attention is given to them. This volume is largely confined to mammalian model systems, in particular human and mouse. It is primarily an excellent state-of-theart overview of mouse neurogenetics, covering advanced methods of genetic manipulation and analysis in forward and reverse genetics.

That said, Methods in Genomic Neuroscience suffers from a few deficiencies. Collating color figures from various chapters and inserting them separately in the book is irritating to the reader, and is a publishing practice of yesteryear. The volume does not cover emerging new methodologies such as single-cell RTPCR, single-cell cDNA libraries and differential display that allow molecular analysis of very small tissue samples and that are thus eminently suitable to the complex and heterogeneous nervous system. And the absence of computational genomics is an inexplicable deficit in a modern treatise of genomic neuroscience.

The potential readership for this volume is very broad: the first-year graduate student, starry-eyed and eager to absorb and embrace it all; the postdoctoral fellow, who may have recently switched fields and needs a primer; the seasoned lab head, who was trained decades ago and may find it difficult to remain up-todate about the new methodologies; the reviewer of grant applications and the science administrator, searching to place projects and proposals in context. Each of these practicioners and facilitators of modern neuroscience will benefit from reading this book. Though a few chapters are somewhat out of line as they are specific and far too detailed, they are easily identifiable and can be skipped by the general reader.

Rather than focusing on one or a few chapters that are of immediate interest to one's research area, as one usually does with a book of this kind, I recommend reading Methods in Genomic Neuroscience in its entirety-from start to finish, or from finish to start, or in any order. That is a compliment that one can rarely offer a book of the methods type. 